IdeAs

Idées d'Amériques

15 | 2020

Eau et gestion de l'eau dans les Amériques

\title{
Les ambivalences de la droite chilienne dans la gestion de la crise
}

\section{Stéphanie Alenda}

\section{(2) OpenEdition}

\section{Journals}

Édition électronique

URL : http://journals.openedition.org/ideas/8379

DOI : 10.4000/ideas.8379

ISSN : 1950-5701

Éditeur

Institut des Amériques

Référence électronique

Stéphanie Alenda, «Les ambivalences de la droite chilienne dans la gestion de la crise », IdeAs [En ligne], 15 | 2020, mis en ligne le 01 mars 2020, consulté le 21 décembre 2020. URL : http:// journals.openedition.org/ideas/8379; DOI : https://doi.org/10.4000/ideas.8379

Ce document a été généré automatiquement le 21 décembre 2020.

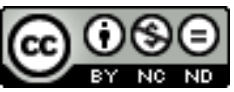

IdeAs - Idées d'Amériques est mis à disposition selon les termes de la licence Creative Commons Attribution - Pas d'Utilisation Commerciale - Pas de Modification 4.0 International. 


\title{
Les ambivalences de la droite chilienne dans la gestion de la crise
}

\author{
Stéphanie Alenda
}

1 En 2010, le Chili élisait démocratiquement son premier président de droite depuis 1958, réélu pour un second mandat en 2017. Les débuts de Sebastián Piñera ont été marqués par son identification avec l'idée d'une " nouvelle droite ", moderne, « très éloignée des totalitarismes et des violations des droits de l'Homme » (El País, 2010), et s'illustrant quelques mois plus tard par l'augmentation des impôts pour financer la reconstruction du pays à la suite du tremblement de terre de février 2010. Lorsque le 19 octobre 2019, le président Piñera a décrété l'état d'urgence en recevant le soutien du leader de la droite radicale, José Antonio Kast ${ }^{1}$, la coalition de centre-droit a paru rebasculer vers son passé autoritaire. Mais quelques jours plus tard, l'invitation du président à différents observateurs des droits de l'Homme (notamment l'ONU et Human Rights Watch), renouait avec l'engagement de leur défense, aspect marquant une ligne de démarcation entre la «nouvelle droite $^{2}$ » et la plus ancienne: l'UDI et certaines fractions de RN ${ }^{3}$.

2 Comment interpréter l'ambivalence de ces prises de position? Pourrait-elle s'expliquer par l'existence de deux droites, une aile dure et l'autre modérée, souvent présentées sous les traits des «faucons" représentants des pouvoirs factuels [poderes de facto] et garants du noyau dur identitaire vs. les "colombes", défenseurs des institutions politiques et œuvrant à la consolidation au pouvoir du secteur (Macari M., 2010 ; Bellolio C., 2016) ? Pour certains, ces deux âmes seraient réapparues sous les traits de l'ancien ministre de l'Intérieur, Andrés Chadwick, dirigeant historique de l'UDI au passé pinochétiste, et Gonzalo Blumel, son successeur, membre d'Evópoli et d'une nouvelle génération de dirigeants (Batarce M.C., 2019). Pour d'autres, la gestion de Blumel ne le différencierait guère de l'aile dure, n'ayant eu aucun impact sur la réduction des violations des droits de l'Homme (Gaete, A. et al., 2019). Ces deux droites auraient-elles une incidence sur la façon dont la crise a été gérée par le gouvernement ?

3 Nous montrerons plutôt que la dynamique de la crise - en particulier le processus constitutionnel -, a révélé l'existence de différentes sensibilités au sein de la droite 
chilienne, cependant unie dans la défense de l'ordre, invitant à complexifier la dichotomie entre les « faucons » et les « colombes».

\section{Le retour aux fondamentaux : l'ordre et l'autorité}

4 Il nous faut tout d'abord replacer cette réflexion dans un cadre général, celui de la sociologie des crises politiques (Dobry M., 1986), qui permet de rappeler que les conjonctures fluides sont particulièrement propices à la radicalisation politique, mais aussi que les ruptures les plus radicales peuvent avoir lieu graduellement, voire dans des configurations dominées à l'origine par des individus modérés ${ }^{4}$. C'est donc dans la dynamique des logiques de situation spécifique qui contraignent perceptions, calculs et tactiques des acteurs qu'il faut comprendre certaines décisions et leurs effets en prenant en compte la façon dont ceux-ci non seulement gèrent mais suscitent et réagissent à certains processus de mobilisation politique, à travers notamment un échange de "coups", c'est-à-dire "d'actes et de comportements individuels ou collectifs » qui affectent « soit les attentes des protagonistes d'un conflit concernant le comportement des autres acteurs, soit ce que Goffman appelle leur "situation existentielle" (...) » (Dobry, M., 1986 : 21). A grands traits, deux moments peuvent être distingués dans la gestion de l'explosion sociale: une phase réactive initiale, la plus critique, succédant à l'incendie simultané de neuf stations de métro qui a conduit le président Piñera à décréter l'état d'urgence, et un second temps où le gouvernement a cherché à avancer sur la voie de la «normalisation », sans effets notoires sur la décrue des mobilisations.

5 La crise a non seulement dévoilé les différentes positions existant au sein de la coalition de gouvernement (nous y reviendrons). Elle a de plus réveillé, par un effet de miroir, certains traumatismes, sans nul doute asymétriques mais non moins rééls, entre une droite qui a ranimé le spectre de l'ennemi interne et de la sécurité nationale à travers le "nous sommes en guerre $»^{5}$ aux accents pinochétistes du président Piñera (20 octobre 2019), hypothéquant par là-même la possibilité d'une résolution politique du conflit ; et une gauche pour laquelle s'est rouverte la plaie des violations des droits de l'Homme en dictature ${ }^{6}$. L'extrême violence des événements interprétée par le gouvernement comme une volonté de « détruire » le Chili et ses valeurs de « liberté et démocratie » mais aussi comme un risque de faillite de l'État où l'auto-défense finirait par être la règle, plus que mettre en évidence l'influence d'une "aile dure» de la droite, a poussé à leur paroxysme les principes constitutifs de son identité : la défense de l'ordre et de l'autorité ${ }^{7}$ avec, en arrière-plan, celle d'un certain modèle de développement ${ }^{8}$. Dans une enquête appliquée en $2015-2016^{9}$, nous avions pu mettre en évidence l'identification massive et transversale à l'ordre des cadres dirigeants de la coalition de gouvernement. A la question: "Les protestations sociales doivent être contrôlées afin de garantir le droit à la libre circulation des personnes ", $84 \%$ de l'UDI, $83 \%$ de RN et $74 \%$ d'Evópoli avaient répondu favorablement. L'enquête montrait aussi l'existence d'une droite se reconnaissant majoritairement $(55,5 \%)$ dans la maxime politique et sociale de subsidiarité, principe clef des politiques néolibérales de la fin des années $70^{10}$.

6 Provocant par une combinaison d'éléments critiques d'ordre décisionnel et institutionnel une sorte de "tempête parfaite ", ces mesures visant à rétablir l'ordre se sont heurtées à la crise de l'échafaudage institutionnel du pays, en particulier celle de l'institution policière. 


\section{Les entraves à la gestion : la crise des institutions}

7 L'incapacité à rétablir l'ordre s'est vue aggravée par la violation massive des droits de l'Homme par les forces de police - 2009 personnes ont été blessées entre le 17 octobre et le 10 novembre, selon l'Institut National des Droits de l'Homme (INDH), dont un nombre important éborgné -, faisant du Chili le pays où l'État exerce la répression la plus forte en temps de conflits sociaux (voir article d'Aguilera dans ce dossier). Ce bilan doit beaucoup à la crise que traverse l'institution policière ${ }^{11}$, dont la réforme a été engagée. Encore considérée par l'opinion publique comme l'une des plus crédibles en 2017, elle figure aujourd'hui parmi les plus décriées, $64 \%$ des Chiliens estimant que les forces de police ont violé les droits de l'Homme depuis octobre 2019 (CEP, 2019). Mais cette violence inusitée - le rapport de l'ONU fait état de 2500 policiers blessés entre le 18 octobre et le 6 décembre - peut être en partie lue comme une réponse à la répression et à la criminalisation de la protestation. Comme le montre la littérature internationale sur d'autres conflits sociaux, les protestataires qui perçoivent que la police a fait un usage indiscriminé de la force à leur encontre tendent davantage à justifier une riposte violente (Maguire, E., et al., 2018). De même, la violence résulte d'interactions se jouant dans des situations particulières où revêt un rôle central une action de la police fondée sur la communication, le respect de certains espaces territoriaux, la reconnaissance du rôle des émotions dans les explosions sociales ou encore le fait d'éviter certains signaux pouvant mener à la surenchère (Nassauer, A., 2014). Au contraire, la stratégie d'endiguement opérationnalisée par la police avec une autonomie beaucoup trop grande vis-à-vis de l'autorité civile n'a fait que creuser l'écart entre citoyens et forces de l'ordre assimilées au gouvernement.

8 Selon le dernier sondage du CEP (2019), les Chiliens condamnent massivement l'usage disproportionné de la force des carabiniers, au même titre qu'ils réprouvent majoritairement l'usage de la violence de la part de certains groupes de manifestants. Ce dernier point, en montrant l'inefficacité du gouvernement dans le rétablissement de l'ordre, a en outre produit une désaffection des bases électorales traditionnelles de la droite à l'égard du gouvernement, reflétée dans le taux d'approbation du président tombé à $6 \%$ (CEP, 2019). En s'inscrivant dans le cadre d'un régime présidentiel exacerbé, ces perceptions constituent une entrave à la gouvernabilité (Cardenas, R., 2020). S'y ajoute le discrédit absolu du Congrès et des partis politiques contribuant à la faible valorisation des avancées en matière d'agenda social, éclipsées par l'omniprésence de l'« agenda sécuritaire ».

9 Autour de cet agenda, rien ne permet donc de conclure à l'existence de deux droites aux convictions contrastées. La droite (au gouvernement, au sein des partis et de l'électorat) semble plutôt se reconnaître dans une position faisant du rétablissement de l'ordre une priorité. Les différences entre les deux ministres de l'Intérieur ayant successivement conduit la gestion de la crise ne tiennent qu'à leurs âges, appartenances partisanes et compétences respectives en matière de gestion et de communication.

10 La dynamique de la crise et le processus constitutionnel ont cependant révélé l'existence de différentes sensibilités au sein de la droite invitant à complexifier la dichotomie entre les « faucons » et les « colombes ». 


\section{Une coalition de centre-droit entre ouverture et repli identitaire}

11 Comme nous l'avons vu, les conjonctures de crise politique et de radicalisation favorisent le retranchement sur un noyau dur identitaire. La phase de «normalisation» a également été marquée par la signature d'un accord transversal pour la paix sociale et la nouvelle Constitution, le 15 novembre 2019, qui a eu pour effet d'apaiser le conflit en répondant à l'aspiration citoyenne d'un nouveau contrat social. Elle a donc eu une répercussion positive - bien qu'indirecte, étant donné que le gouvernement est demeuré en marge des négociations - sur la gestion de la crise. Mais très vite, l'accord, arraché sous la contrainte de la rue ${ }^{12}$, s'est défait à la suite de la présentation par l'opposition, à l'initiative du Parti Communiste et du Frente Amplio ${ }^{13}$, d'une série d'accusations constitutionnelles visant à responsabiliser les plus hautes autorités de l'État pour les violations des droits de l'Homme. Ces signaux contraires à l'engagement initial pour le rétablissement de l'ordre, s'ajoutant aux violences de rue, ont fini par réveiller au sein de la droite le spectre de l'Unité Populaire et cristalliser l'opposition à une nouvelle Constitution. Pour le sénateur Allamand, l'un des représentants les plus influents de l'option du rejet, le processus constitutionnel s'est ainsi converti en une "opportunité de ranimer un centre-droit vacillant (...) en défendant sans complexe les idées de la droite face à la gauche radicale » (Valenzuela, P., 2020).

12 La crise a ainsi mis à nu l'existence de deux positions au sein de la droite, considérées l'une comme l'autre légitimes, c'est-à-dire sans effet polarisant autour du clivage autoritarisme/démocratie (Muñoz, A., 2020). La première, majoritaire, va dans le sens des réponses recueillies dans le cadre de notre enquête où $79 \%$ de l'UDI et $65 \%$ de RN se déclaraient contraires à l'établissement d'une nouvelle Constitution, tandis que seuls 39 \% d'Evópoli exprimaient leur désaccord avec ce changement, confirmant au sein du conglomérat l'existence d'une "nouvelle droite " nettement dissociée de l'héritage pinochétiste (Alenda, S. et al., 2020b). La seconde, minoritaire, réunit un groupe important de cadres dirigeants mais également certaines figures clef de la coalition présentes même dans les formations politiques s'inclinant pour le Non ${ }^{14}$. Elle coincide avec l'opposition sur d'autres sujets tels que la défense d'une participation égalitaire des femmes et des hommes au sein de la convention constituante.

Plus que l'existence de « faucons » et de « colombes » que différencierait le rapport aux institutions démocratiques, le centre-droit actuel renferme des sensibilités différentes, parfois croisées, les unes davantage garantes du noyau dur identitaire (conservateur sur le plan des mœurs, subsidiaires quant au rôle attribué à l'État dans l'économie) que les autres (plus libéraux et solidaires). En fonction des « coups » auxquels se livreront les différents acteurs du conflit avant la tenue du plébiscite, il faudra s'attendre à un renforcement de la tendance à la réaction identitaire ${ }^{15}$, de même qu'à une augmentation croissante du rejet à une nouvelle Constitution ou encore à la victoire de la convention mixte (Retamal P., 2020). Dénouement éventuel bien éloigné de l'accord interpartisan du 15 novembre pour une nouvelle Constitution. 


\section{BIBLIOGRAPHIE}

Alenda, Stéphanie, « Les avatars de la " nouvelle droite " chilienne : la fabrique d'une institution partisane (1967-2010) », POLITIX. Revue des sciences sociales du politique, no106 (27), 2014, p. $135-161$.

Alenda, Stéphanie et al., « Evolución Política y la renovación liberal en Chile ». Anatomía de la derecha chilena : Estado, mercado y valores en tiempos de cambio, Santiago, Fondo de Cultura Económica, 2020a, p. 157-189.

Alenda, Stéphanie et al., « Evolución de las sensibilidades políticas : Hacia una nueva centroderecha en Chile ». Anatomía de la derecha chilena : Estado, mercado y valores en tiempos de cambio, Santiago, Fondo de Cultura Económica, 2020b, p. 87-118.

Alenda, Stéphanie et al., « El estudio de las élites políticas en Chile : figuras y sostenes del orden ». Las élites políticas en el Sur. Un estado de la cuestión de los estudios sobre la Argentina, Brasil y Chile, Buenos Aires, éditions UNGS, 2018, p. 153-205.

Batarce, María Catalina, « Las renuncias que señalan el segundo tiempo del Gobierno », Pauta, 18 décembre 2019, https://www.pauta.cl/politica/las-renuncias-que-senalan-el-segundo-tiempodel-gobierno, page consultée le 24 janvier 2020.

Bellolio, Cristóbal, « Halcones y palomas : la derecha frente al proceso Constituyente ", revista Capital, 14 mai 2016. https://politicaparaprincipiantes.com/2016/05/14/halcones-y-palomas-laderecha-frente-al-proceso-constituyente/ Page consultée le 22 janvier 2020.

Cardenas, Rodrigo, «Se buscan los votantes de Piñera : sólo el 18 \% dice que votó por él en segunda vuelta y mayoría de la derecha lo desaprueba », La Tercera, 24 janvier 2020, https:// www.latercera.com/la-tercera-pm/noticia/se-buscan-los-votantes-de-pinera-solo-18-dice-quevoto-por-el-en-segunda-vuelta-y-mayoria-de-la-derecha-lo-desaprueba/985664/, page consultée le 23 janvier 2020.

Collovald, Annie et Gaiti, Brigitte, La démocratie aux extremes. Sur la radicalisation politique, Paris, La Dispute, 2006.

Dobry, Michel, Sociologie des crises politiques, Paris, Presses de Sciences Po, 1986.

Roy Calvo, Jose Manuel, « Sebastián Piñera : "Estamos construyendo una nueva derecha" », El País, Madrid, 7 novembre 2010, https://elpais.com/diario/2010/11/07/internacional/ 1289084402_850215.html, page consultée le 24 janvier 2020 .

Encuesta CEP, Estudio Nacional de Opinión Pública, n84, décembre 2019. www.cepchile.cl, page consultée le 9 mars 2020

Gaete, Ayleen et al., " 60 días de violencia en el Oasis imaginario », CIPER/Académico, 19 décembre 2019, https://ciperchile.cl/2019/12/19/60-dias-de-violencia-en-el-oasis-imaginario/, page consultée le 24 janvier 2020.

Le Foulon, Carmen et al., " Metodología de la encuesta a dirigentes de Chile Vamos ». Anatomía de la derecha chilena: Estado, mercado y valores en tiempos de cambio, Santiago, Fondo de Cultura Económica, 2020, p. 347-369.

Macari, Mirko, «Halcones, palomas y la encrucijada dialéctica de la derecha », El Mostrador, 8 avril 2010, https://www.elmostrador.cl/noticias/pais/2010/04/08/halcones-palomas-y-laencrucijada-dialectica-de-la-derecha/, page consultée le 9 mars 2020 
Maguire, Edward et al., «Attitudes towards the Use of Violence against Police among Occupy Wall Street Protesters », Policing, 2018, p. 1-17.

Muñoz, Andrés, «Stéphanie Alenda, socióloga : no veo que el proceso constituyente lleve a una crisis terminal de Chile Vamos », La Tercera, 9 janvier 2020, https://www.latercera.com/latercera-pm/noticia/stephanie-alenda-sociologa-no-veo-proceso-constituyente-lleve-una-crisisterminal-chile/967229/, page consultée le 23 janvier 2020.

Nassauer, Anne, « Effective crowd policing: empirical insights on avoiding protest violence », Policing: An International Journal of Police Strategies \& Management, vol. 38, n1, 2015, p. 3-23.

Retamal, Pablo, « Cadem : Baja respaldo al “Apruebo” en el plebiscito y hay “empate técnico" entre convención totalmente electa y fórmula mixta ", La Tercera, 26 janvier 2020, https:// www.latercera.com/politica/noticia/cadem-baja-respaldo-al-apruebo-plebiscito-empate-tecnicoconvencion-totalmente-electa-formula-mixta/987800/, page consultée le 23 janvier 2020.

Valenzuela, Paula, Andrés Allamand, senador de Renovación Nacional : « Nuestra postura ya no tiene vuelta atrás », El Mercurio, 12 de enero de 2020, https://digital.elmercurio.com/2020/01/12/ R/JU3NVR71\#zoom =page-width, page consultée le 23 janvier 2020.

\section{NOTES}

1. José Antonio Kast s'est présenté comme candidat indépendant aux élections présidentielles de 2017, arrivant en quatrième position avec 7,9\% des suffrages exprimés, après une campagne visant à conquérir l'aile dure et conservatrice de la droite.

2. Par cette catégorie, nous nous référons non seulement à la «nouvelle droite » du premier mandat de Sebastián Piñera mais aussi à Evópoli, fondé en 2015 comme un projet de rénovation de la droite chilienne. Pour plus de détails sur ce parti, nous renvoyons à Alenda S. et al., 2020a.

3. L'Union Démocrate Indépendante est un parti politique issu du mouvement conservateur dit "grémial » fondé en 1987 ayant fourni de nombreux cadres à l'administration de la dictature militaire du général Augusto Pinochet (pour plus de détails, voir Alenda S., 2014). Rénovation Nationale est fondé à la même date comme un parti de centre-droit qui constitue à l'heure actuelle l'aile modéréé de la coalition de gouvernement Chile Vamos.

4. Nous renvoyons sur ce sujet à l'ouvrage collectif dirigé par Annie Collovald et Brigitte Gaiti, 2006.

5. Gonzalo Cordero, militant historique de l'UDI et directeur de communication de la seconde campagne de Sebastián Piñera considère cette phrase comme une grave erreur de communication car elle a contribué à la stigmatisation de la protestation sociale. Elle s'explique selon lui par un contexte où le Président venait d'être informé de tentatives de «sabotage du réseau de communication et de piratage du système financier du pays ». D'après Cordero, «le problème du contrôle de l'ordre public au stade où nous étions posait un problème de gouvernabilité, un problème de stabilité du gouvernement. Nous avons été sur le point de franchir un seuil après lequel le gouvernement n'existait simplement plus. Il y avait donc une nécessité tant symbolique que matérielle de récupérer l'ordre public, le contrôle du territoire au sens de l'espace public », entretien à Santiago le 16 janvier 2019.

6. Comment interpréter autrement la polémique, au début de l'état d'urgence, sur l'existence d'un centre de torture dans une station de métro de Santiago ou encore celle de personnes disparues. Bien que non avérées, ces rumeurs ont été relayées par les commentateurs de l'actualité chilienne.

7. Alenda S. et al., 2018, montrent cependant que l'obsession pour ces principes transcende au Chili la culture politique « de droite». 
8. Entretien avec Gonzalo Cordero, Santiago, 16 janvier 2019.

9. Pour plus de détails, voir Le Foulon C. et al., 2020.

10. Elle puise son origine dans la doctrine sociale de l'Église qui circonscrit l'intervention de l'État aux situations où les particuliers ou groupes intermédiaires sont démunis des compétences nécessaires pour se suffire à eux-mêmes dans leurs domaines respectifs. Voir Alenda S. et al. $2020 b$.

11. Les malversations de fonds et abus de pouvoir en tout genre ont précipité la débâcle au sein de l'institution, décimée d'une quarantaine d'officiers entre décembre 2017 et décembre 2018.

12. Selon Gonzalo Cordero, la droite s'est trouvée face à deux alternatives : la signature de cet accord ou la démission du président Piñera. La seconde comportant davantage de risques d'instabilité politique, c'est la première qui a été retenue. Entretien à Santiago le 16 janvier 2019.

13. Le Frente Amplio est une coalition composée de partis et de mouvements politiques de gauche et d'extrême-gauche.

14. Joaquín Lavín, le maire UDI de l'une des communes les plus riches du grand Santiago, Las Condes ; le sénateur Manuel José Ossandón de RN et le président de ce parti, Mario Desbordes.

15. Plusieurs épisodes récents ont eu cet effet, parmi lesquels l'ovation faite aux « héros » de la "première ligne» (manifestants légitimant l'usage de la violence) lors d'un récent Forum latinoaméricain sur les droits de l'Homme tenu le 24 janvier 2020 au sein de l'ex Congrès national et organisé par un groupe de sénateurs de l'opposition. Y ont participé les ex présidents Evo Morales et Rafael Correa, mais également le juge Baltasar Garzón qui avait joué un rôle clé dans la détention d'Augusto Pinochet.

\section{AUTEUR}

\section{STÉPHANIE ALENDA}

Stéphanie Alenda est affiliée à l'Université Andrés Bello - FECS

salenda@unab.cl 